\title{
Alongamento
}

\section{Alongamento Muscular Descompensado e Postura}

\section{Stefano Piloni}

Programa de Pós-Graduação em Posturalogia - Primeira Faculdade de Medicina e

Cirurgia - Dept ${ }^{\circ}$ de medicina Esperimental e Patologia - Universidade dos Estudos "La

Sapienza" de Roma

stefpiloni@yahoo.com

\section{José Guilherme Fernandes Bertoni da Silva}

Universidade da Coruña - UDC Espanha

igbertoni@virgilio.it

\section{Xurxo Dopico}

Universidade da Coruña - UDC - Espanha

dopico@udc.es

PILONI, S; SILVA, J.G.F.B.; DOPICO, X. Alongamento muscular descompensado e postura. Fitness \& Performance Journal, v.5, $\mathrm{n}^{\circ}$ 6, p. 344-347, 2006.

RESUMO - A correta amplitude fisiológica do movimento, relativa a cada articulação, depende de uma boa relação articular e de um bom equilíbrio das tensões. $\bigcirc$ estudo foi realizado com 24 indivíduos portadores de problemas posturais adaptativos, com idade entre 40 e 70 anos, divididos de forma não-probabilística em três grupos. No Grupo 1 foi aplicado o "método Raggi" utilizando a Pancafit $\AA$ The Original ( $N=8)$; o Grupo 2 realizou exercícios calistênicos de baixa intensidade a corpo livre, com alongamento muscular tradicional - "Método Bob Anderson" ( $N=8)$; e o Grupo 3, grupo controle, não sofreu intervenção alguma, nem mesmo medicamentosa $(\mathrm{N}=8)$. Hipertensão arterial, flebite, doenças oncológicas, escoliose ortopédica e cirúrgica $\left(>\mathrm{de} 25^{\circ} \mathrm{COBB}\right)$, osteoporose em estado avançado, vestibolopatia, demência senil e problemas neurológicos foram considerados critério de exclusão. Os resultados obtidos por esta pesquisa demonstraram como a Pancafit ${ }^{\circledR}$ e o "Método Raggi ${ }^{\circledR}$ " produzem importantes alterações na postura, com uma tendência ao realinhamento da coluna vertebral, aproximando-se dos parâmetros fisiológicos ideais.

Palavras-chave: alongamento, articulação, postura

\section{Endereço para correspondência:}

Rua André Rocha, 3215 sl 207 Curicica - Rio de Janeiro/RJ CEP: 22710-560

Copyright@ 2006 por Colégio Brasileiro de Atividade Física Saúde e Esporte. 


\section{Muscular Allonge Descompensado And Position}

The correct physiological amplitude of the movement, relative to each joint, depends on a good relation to articulate and a good balance of the tensions. The study it was carried through with 24 carrying individuals of adaptativos posturais problems, with age between 40 and 70 years, divided of not-probabilist form in three groups. In Group 1 the "Raggi method" was applied using the Pancafit $\AA$ Original The ( $\mathrm{N}=8)$; Group 2 carried through calistênicos exercises of low intensity the free body, with traditional muscular allonge - "Method Bob Anderson" $(\mathrm{N}=8)$; e Group 3, group has controlled, did not suffer to intervention some, not even medicamentosa $(\mathrm{N}=8)$. Arterial Hipertensão, oncológicas flebite, illnesses, escoliose ortopédica and surgical (of $25^{\circ} \mathrm{COBB}$ ), osteoporose in advanced state, vestibolopatia, senile dementia and neurological problems had been considered exclusion criterion. The results gotten for this research had demonstrated as the Pancafit ${ }^{\circledR}$ and the "Raggi ${ }^{\circledR}$ Method" produces important alterations in the position, with a trend to the realinhamento of the vertebral column, coming close itself to the ideal physiological parameters.

\section{Allonge Muscular Descompensado Y Posición}

La amplitud fisiológica correcta del movimiento, concerniente a cada empalme, depende de una buena relación para articular y de un buen equilibrio de las tensiones. El estudio fue llevada a través con 24 individuos que llevaban de los problemas de los posturais de los adaptativos, con edad entre 40 y 70 años, divididos de forma del no-probabilista en tres grupos. En el grupo 1 el "método de Raggi" fue aplicado usando la original de Pancafit ${ }^{\circledR}(\mathrm{N}=8)$; El grupo 2 llevó con ejercicios de los calistênicos de la intensidad reducida el cuerpo libre, con el allonge muscular tradicional - "método Bob Anderson" ( $\mathrm{N}=8)$; el grupo 3, grupo de e ha controlado, no sufrió a la intervención alguno, medicamentosa no uniforme $(\mathrm{N}=8)$. Hipertensão arterial, el flebite de los oncológicas, las enfermedades, el ortopédica del escoliose y quirúrgico (de $25^{\circ} \mathrm{COBB}$ ), osteoporose en estado avanzado, vestibolopatia, demencia senil y los problemas neurológicos habían sido considerados criterio de la exclusión. Los resultados conseguidos para esta investigación habían demostrado como el Pancafit ${ }^{\circledR}$ y el "método de Raggi ${ }^{\circledR}$ " produce alteraciones importantes en la posición, con una tendencia al realinhamento de la columna vertebral, viniendo cerca sí mismo a los parámetros fisiológicos ideales.

Palabras-clave: allonge, empalme, posición

\section{INTRODUÇÃO}

A correta amplitude fisiológica do movimento, relativa a cada articulação, depende de uma boa relação articular e de um bom equilíbrio das tensões que os músculos produzem sobre a mesma (Léopold Busquet, 2002). Agindo em um esquema postural desequilibrado, através de uma técnica de alongamento global com a finalidade de restituir a elasticidade, o comprimento e a simetria das cadeias musculares, o resultado esperado seria o reequilíbrio do esquema postural e da boa relação mio-articular.

\section{OBJetivo}

A presente investigação trata de um método de alongamento global denominado "Método Daniele Raggi $\AA^{\circledR}$ ", o qual se baseia no destensionamento mio-articular global. Tal método visa o realinhamento postural e a diminuição da dor. Desta forma, o objetivo desta pesquisa é verificar a eficácia do "Método Raggi" (com o instrumento Pancafit $\circledast$ The Original) nas variações da postura em relação aos parâmetros monitorados, buscando o realinhamento postural (aproximando-se aos parâmetros ideais de postura) e a diminuição da dor nos pontos em que era presente.

\section{MATERIAL E MÉTODOS}

\section{Amostra}

O presente estudo foi realizado com 24 indivíduos portadores de problemas posturais adaptativos, com idade entre 40 e 70 anos. Foram divididos de forma não-probabilística em três grupos, sem distinção de sexo ou nível social, escolhidos independentemente do fato de sentirem dores (a menos que se encontrassem em fase aguda). No Grupo 1 foi aplicado o "método Raggi" utilizando a Pancafit ${ }^{\circledR}$ The Original $(N=8)$; o Grupo 2 realizou exercícios calistênicos de baixa intensidade a corpo livre, com alongamento muscular tradicional - "Método Bob Anderson" (N=8); e o Grupo 3 , grupo controle, não sofreu intervenção alguma, nem mesmo medicamentosa $(\mathrm{N}=8)$. Considerou-se como critério de exclusão qualquer tipo de condição aguda ou crônica que estivesse fora dos propósitos do presente estudo, tais como: hipertensão arterial, flebite, doenças oncológicas, escoliose ortopédica e cirúrgica (> de $\left.25^{\circ} \mathrm{COBB}\right)$, osteoporose em estado avançado, vestibolopatia, demência senil e problemas neurológicos.

Os participantes desta pesquisa assinaram o termo de consentimento, e os procedimentos experimentais foram executados dentro das normas éticas previstas na Resolução No 196, de 10 de Outubro de 1996, do Conselho Nacional de Saúde.

O estudo teve seu projeto de pesquisa submetido e aprovado pelo Comitê de Ética em Pesquisa Envolvendo Seres Humanos da Universidade de Roma, Itália.

\section{Procedimento}

A pesquisa foi desenvolvida em três fases:

$\left.1^{a}\right)$ Teste preliminar (pré-teste), que consistiv em um exame postural no qual foram avaliados: a) a estática em posição ereta nos três planos espaciais, com um exame clássico de base do sistema tônico postural (B. BRICOT, 1998) e a vertical de Barrè no plano frontal (M. ZANARDI, 1998); b) a elasticidade da cadeia muscular posterior com o teste de sidebending anterior (KENDALL F.P., PROVANCE P.G.); c) as assimetrias direita/esquerda do sistema extrapiramidal, com o teste de marchar no mesmo lugar de Unterberger (G. GUIDETTI e D. MARCHIONI, 2002); d) a dor através da escala analógica visível (VAS).

$2^{a}$ ) Protocolo de treinamento especifico para cada grupo. 
$\left.3^{a}\right)$ Teste final (pós-teste), baseado no mesmo exame postural do teste inicial.

A avaliação dos desvios, em relação ao exame postural, foi feita através do critério de atribuição de valores, considerando as medidas em centímetros $(\mathrm{cm})$ e em graus (o). Estas foram reveladas pela diferença entre o valor do parâmetro postural ideal e o valor obtido nos testes realizados. O Grupo 1 realizou o treinamento uma vez por semana, por 45 minutos; o Grupo 2 fez o treinamento duas vezes por semana, por 60 minutos (40 minutos de exercícios calistênicos e 20 minutos de exercícios de alongamento tradicional); e o Grupo 3 não realizou nenhum treinamento.

\section{RESULTADOS}

1) A Figura 1 apresenta o diagrama com as variações percentuais entre a soma dos valores do teste preliminar e a soma dos valores do teste final, que se distanciaram do fisiológico ideal, encontrado em todos os sujeitos dos três grupos. Foram analisadas nos três grupos as variações ocorridas e a média na capacidade de rotação da cabeça para a direita e para a esquerda. Entre os três grupos, o Grupo 1 foi o que obteve maiores ganhos no realinhamento postural e na rotação da cabeça, tanto para a direita como para a esquerda.

2) Na rotação da cabeça, além do aumento do grau de mobilidade, foi evidenciado um reequilíbrio entre os valores da rotação para a direita e para a esquerda no pós-teste. Na Figura 2, podese verificar que o Grupo 1 obteve resultados mais significativos que os demais, pois apresentou um maior número de pessoas com rotação simétrica no teste final.

3) A Figura 3 dispõe da análise comparativa das variações percentuais para a vertical de Barrè. Podem ser observadas, com maior evidência, variações significativas obtidas pelo Grupo 1, quando comparadas aos demais grupos.

4) Na avaliação do teste de Sidebending anterior, disposta na Figura 4, foram comparadas, nos três grupos, as variações percentuais da flexibilidade direita, esquerda e a média entre as duas. Na análise comparativa entre os grupos, o Grupo 1 revelou um maior aumento percentual na flexibilidade bilateral e na média entre os dois lados.

5) Além de ter conseguido um aumento no grau de flexibilidade, o Grupo 1 foi o que obteve um retorno da simetria no pós-teste, fato que não ocorreu nos outros dois grupos. Na Figura 5 pode-se observar que, no teste preliminar, 4 das 8 pessoas do Grupo 1, tinham um grau de flexibilidade simétrica em ambos os lados; no teste final, todos os sujeitos alcançaram esse resultado. Entretanto, no Grupo 2 e no Grupo 3 não houve variações.

6) A Figura 6 apresenta o gráfico com as variações percentuais entre a soma dos valores do pré e pós-teste, as quais se distanciaram do 0 fisiológico ideal encontrado em todo os indivíduos dos três grupos. No teste de Unterberger os valores obtidos se apresentaram contrastantes e não lineares com os valores obtidos no teste preliminar. Provavelmente, isso se deve às diversas variáveis intervenientes internas (aparelho dental, aparelho vestibular, aspecto emocional...) e externas. No Grupo 1 nota-se que houve uma redução da rotação, com uma variação de $8 \%$ e uma redução dos movimentos ântero-posterior (variação de 17\%), e latero-lateral (-103\%). O Grupo 2 teve uma variação de $9 \%$ nas rotações, com diminuição dos movimentos latero-lateral (-38\%) e
Figura 1

Variaçōes percentuais obtidas na rotação bilateral da Cabeça

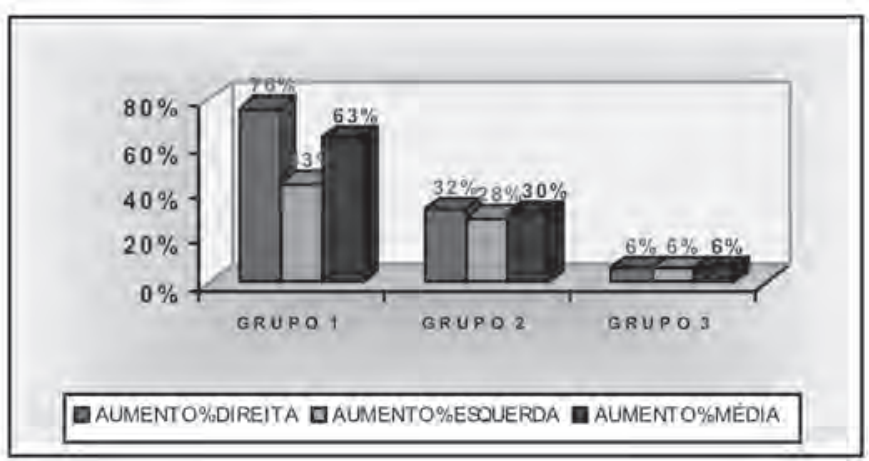

FIGURA 2

Comparativo entre o pré e pós-teste do número de indivíduos QUE OBTIVERAM O REEQUILÍBRIO NA ROTAÇÃo DA CABEÇA

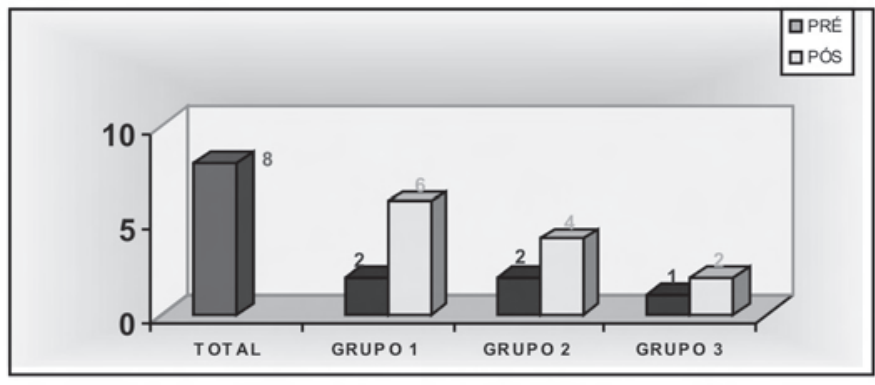

Figura 3

Variações percentuais obtidas na Vertical de Barrè

\section{VERTICAL DE BARRĖ: Comparação das} variações $\%$ entre os grupos

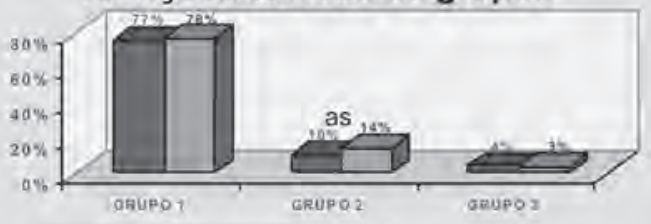

IMEDIA \% Q VALORMEDIO

ântero-posterior (12\%). No Grupo 3 foi encontrada uma variação de $-71 \%$ nas rotações, com redução de $15 \%$ no movimento latero-lateral e de 13\% no movimento ântero-posterior.

7) $\bigcirc$ último parâmetro avaliado foi a variação da dor. Na Figura 7 encontra-se a análise comparativa das variações percentuais. Percebe-se uma diferença significativa nas variações obtidas pelo Grupo 1 em relação ao Grupo 2 e, ainda maior, quando comparada ao Grupo 3. No Grupo 1, 7 dos 8 sujeitos queixavam-se de um ou mais sintomas dolorosos avaliados através da escala analógica visível (VAS) no teste preliminar, sendo verificadas variações percentuais significativas no pós-teste. A soma dessas variações definiu o valor médio global do grupo (média \%).

$\bigcirc$ sujeito 1 reclamava de dor na região lombar, com um índice de 60 no teste preliminar, o qual diminuiu para 15 no teste final, e também de dor na parte esquerda do quadril, com um índice de 70, o qual permaneceu invariado.

○ sujeito 2 apresentava dor na região dorsal, com índice de 40, que desceu para 15; além de dor na região lombar, com um índice de 40, que permaneceu inalterado. 
Figura 4

Variações percentuais obtidas na flexibilidade bilateral da cabeça SEgundo o teste de Sidebending anterior

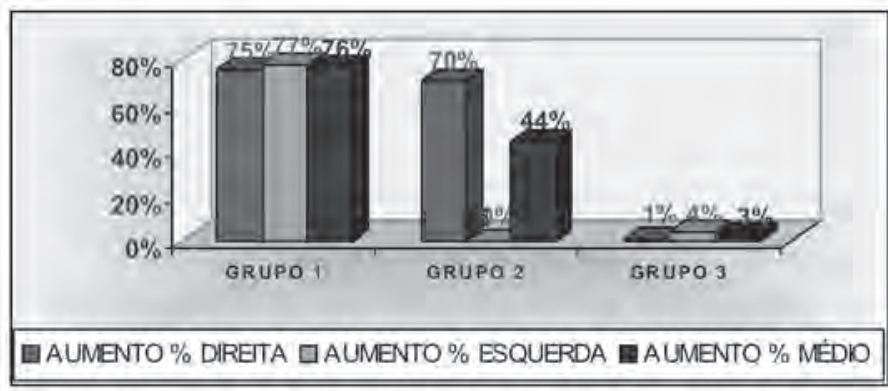

FIGURA 5

Comparativo ENTRE O PRÉ E PÓS-TESTE do NúMERO dE INDiVÍDUOS QUE OBTIVERAM A SIMETRIA NA FLEXIBILIDADE DA CABEÇA, AVALIADA SEGUNDO SIDEBENDING

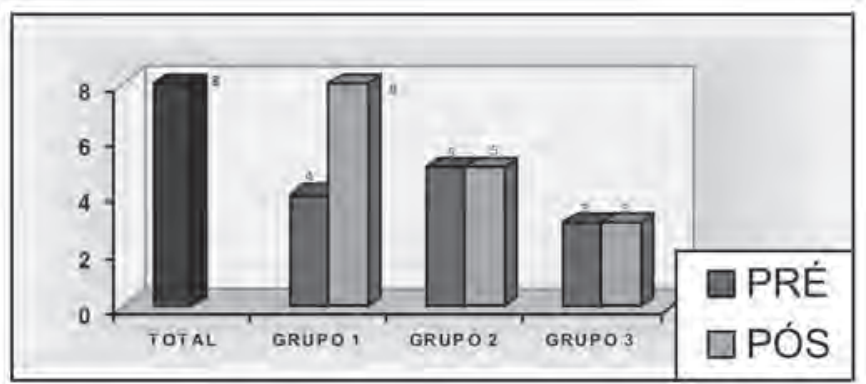

Figura 6

Variações percentuais entre a soma dos Valores pré e pós-teste SEGUNDO O TESTE DE UNTERBERGER

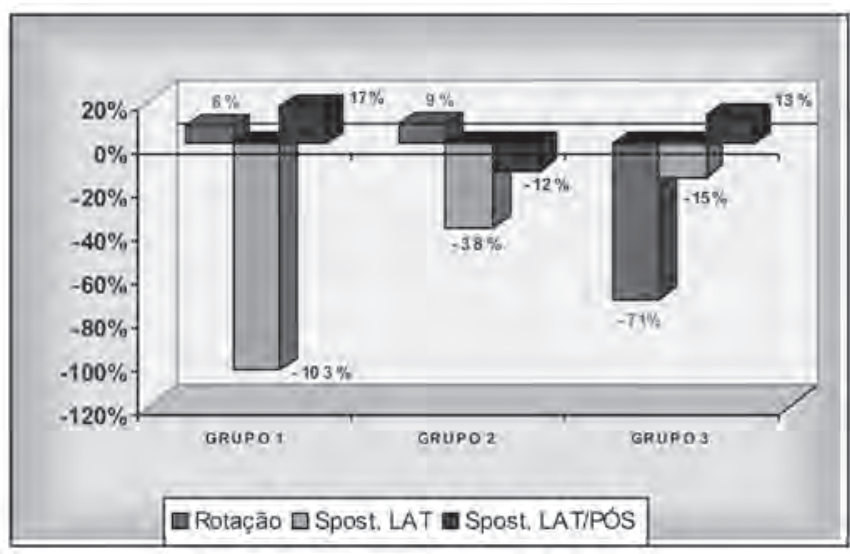

FIGURA 7

VARIAÇÃo percentual obtida No Parâmetro da dor

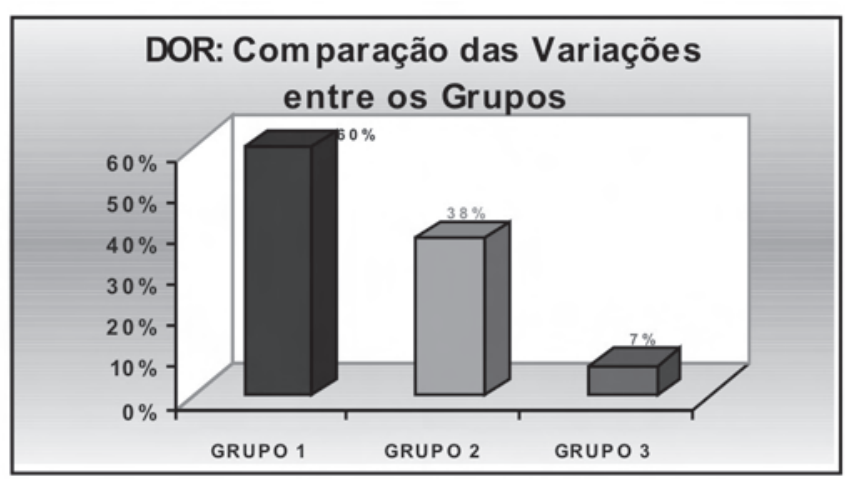

○ sujeito 3 relatava uma dor que partia da região cervical até o ombro, com uma intensidade de 60, a qual foi reduzida a 30 . mesmo indivíduo descrevia dor no pulso direito avaliada com o índice de 100, que se manifestava 5 vezes ao dia e com duração de alguns minutos a cada vez. No pós-teste foi observada uma diminuição da intensidade para 70 e da freqüência para aproximadamente 2 vezes ao dia.

○ sujeito 4 queixava-se de forte tensão cervical, que permanecia presente o dia inteiro, avaliada com uma intensidade de índice resentavam queixa álgica. Essa reflexão não é válida para o Grupo 1, no qual 7 dos 8 sujeitos apresentavam pontos dolorosos.

O único parâmetro que discorda dos valores precedentes monitorados foi o teste de Unterberger, no qual os valores obtidos foram contrastantes e não diretamente lineares com os precedentes.

Provavelmente, isso pode ter occorrido devido ao grau de sensibilidade deste teste, pois existem inúmeras variáveis intervenientes que contribuem para condicionar o resultado final. Essas variáveis podem ser intrínsecas ao proprio sujeito ou extrínsecas. Por esses motivos, não foi encontrada uma linha lógica nos resultados obtidos.

Os autores ressaltam ainda que, neste método (derivado de Mézières), a observação e a experiência são fatores que condicionam extremamente o resultado final.

Em conclusão, pôde-se demonstrar que o Método "Raggi", associado ao aparelho Pancafit $\AA$, se mostrou mais eficiente que o método utilizado no Grupo 2, alcançando melhor realinhamento postural e recuperação da simetria. Entretanto, o Grupo 3 não obteve nenhuma variação significativa.

\section{REFERÊNCIAS BIBLIOGRÁFICAS}

ANDERSON B. Stretching Ed.Mediterranee, 2001.

BRICOT B. La riprogrammazione posturale globale. Ed. Statipro, Marseille (Francia), 1998.

BUSQUET L. Le catene muscolari. Vol. I - IV, Ed. Marrapese, Roma, 2002.

DENNYS -STRUIF G. II manuale del Mezierista. Vol. I - II, Ed. Marrapese, Roma, 1996. GUIDETTI G. e MARCHIONI D. Vestibolo e sport. Ed. Martina, Bologna, 2002.

KAPANDJI I. A. Fisiologia articolare. Ed. Monduzzi, 1996.

KENDALL F.P.; PROVANCE P.G. I. Muscoli funzione e test, quarta edizione con posture e dolore. Ed.Verducci.

VERKIMPE-MORELLI N. e BIENFAIT M. Armonizzazione statica globale. Metodo delle tre squadre. Ed. Marrapese, Roma, 1991.

ZANARDI M. Posturologia clinica osteopatica Ed. Marrapese, Roma, 1998.

Articoli tecnico scientifici, dalla rassegna stampa di Studio Sport 2000, 2003:

Wellback, memorie muscolari, posture e algie.

Traumi, atteggiamenti antalgici ed alterazioni posturali.

L'ernia discale come manifestazione di un processo compressivo di origine muscolare.

Nuova modalità di recupero dell'insufficienza respiratoria.

Allungamento muscolare globale decompensato in relazione alla postura.

Una proposta per "allontanare" la protesi.

Articoli tecnico scientifici di F. SCOPPA, da Otoneurologia 2000, 2002:

- Posturologia: il modello neurofisiologico, il modello biomeccanico, il modello psicosomatico. 